\title{
Intérêt de la méthode historique pour les modèles réduits à fond mobile
}

\author{
APPLICATION A L'ESTUAIRE DE LA SEINE
}

\author{
Advantages of the "historical" method \\ in movable bed model testing
}

\author{
Application to the Seine estuary
}

PAR JEAN CHAPON

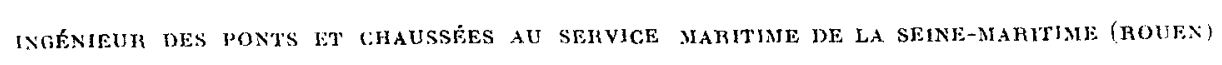

\begin{abstract}
Difficulté de l'étude de l'aménagement d'un estuaire à fond mobile en raison de la maltiplicité des facleurs conditionnant l'évolution des profondeurs, et de l'ignoranee des lois de transports des matériaux solides.

Essai d'évolntion historique tentí sur le modèle réduit de l'estuaire de la Seine pour reproduire l'évolution des profondeurs dans le passé. Concordance entre les fonds du modele et ceux de la nature, pour une période comprise entre 1875 et 1953, permeltant de vérifier la justesse, la sensibilité et la fidélité du modèle. Définition de l'échelle des temps d'évolution des fonds qui ne pouvait être déterminée par les considérations théoriques. Le succès des essais dn "passé» a permis de faire confiance à ceux de l'avenir.

Intérêt de la vérification de la concordance des profondeurs d'un modèle réduit avec celles de la nature pendant une longue période du passé pour l'étude rationnelle de phénomènes complexes régis par des facteurs très nombrenx.
\end{abstract}

\begin{abstract}
Mabile-bed estucry development studies are difficult to undertake because of the very many factors governing bed evolution and a lack of knowledge of solid transport relationships. An attempt was made on the scale model of the Seine estuary to reproduce past bed developments. The model results agreed well with the real-life trends recorded between 1875 and 1953, thus confirming that the model was accurate, sensitive and representative. A time scale for the bed developments was thus defined, which could not have been determined by a theoretical approach. Having reproduced past events to within a high degree of accuracy, the model could also be expected to provide a reliable representation of future trends.

Such confirmed agreement between water depths on a model and in real life over a long period of years is valuable for a rational study of complex phenomena governed by " very large number of factors.
\end{abstract}

\section{1. - LES ETUdeS SUR MODËLE RÉdUIT DE L'ESTUAIRE DE LA SEINE}

1.1. - LE PROBLÈME POSÉ PAR L'ESTUAIRE DE IAA SEINE (fig. 1). - Les problèmes d'exploitation du port de Rouen sont dominés, depuis plusieurs siècles, par celui du tirant d'eau maximal autorisé par ses accis et en particulier par l'estuaire de la Seine dont le chenal est instable et peu profond.

Une loi du 11 janvier 1932 a approuvé un avant-projet d'aménagement consistant à établir dans l'estuaire de la Seine un chenal stable et 


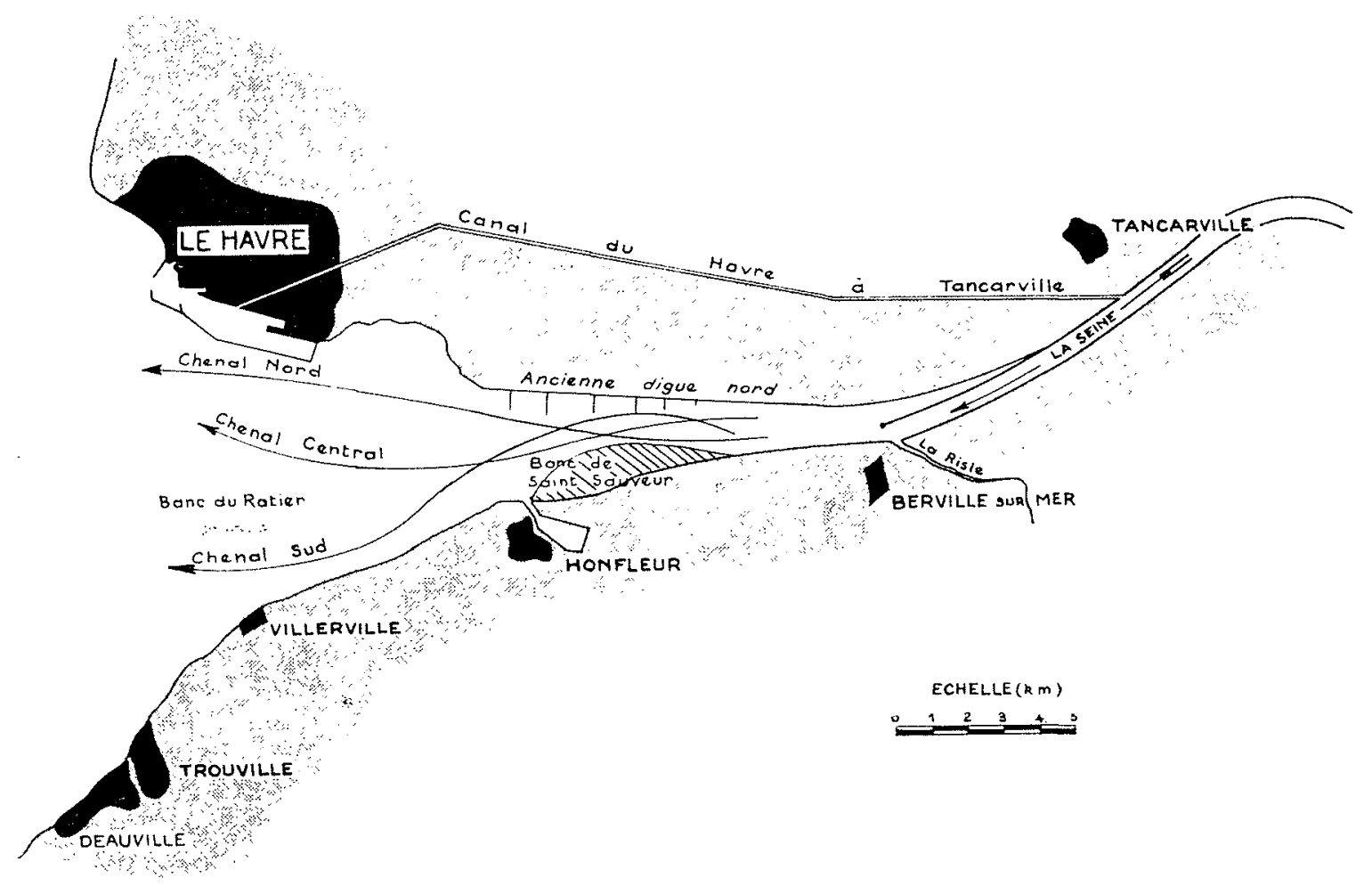

Fia. 1

Plan schématique de l'estuaire de la Seine

(avant exécution des travaux de la loi du 11-1-32).

régulier, dont les profondeurs soient supérieures de 2 à $3 \mathrm{~m}$ aux valeurs actuelles.

Avant d'exécuter des travaux, donl le coût avoisine une vingtaine de milliards d'anciens franes (valeur 1959), le Port de Rouen a voulu s'entourer de garanties suffisantes sur la réussite du projet et a engagé de très importants essais sur modèle réduit au Laboratoire de la Société Neyrpic à Grenoble, devenue depuis la SOGREAH. C'est au cours de ces essais que fut appliquée la méthode historique pour déterminer les éléments que les réglages les plus minutieux n'avaient pas permis d'obtenir.

1.2. - LA NÉCESSITÉ DES ESSAIS SUR MODÈLE RÍDurT. - Très schématiquement, l'aménagement de l'estuaire de la Seine consiste à raccorder de façon régulière la partie fluviale endiguée située en amont de la Risle, à la partie maritime de la Baie de Seine, par une zone de transition oì les conditions d'écoulement hydraulique et de débit solide trouvent un état d'équilibre stable.

L'étude théorique du problème ne pouvait se concevoir avec une rigueur mathématique, en raison du grand nombre et de la complexité des phénomènes naturels (marée, houle...), de leur caractère régulier ou aléatoire et de l'ignorance des lois physiques.

L'observation des phénomènes naturels et le résultat des aménagements exécutés avant celui de la loi de 1932 ne permettaient pas non plus d'en tirer des conclusions valables pour décider d'un nouvel aménagement.

Il était donc logique d'envisager la construction et l'exploitation d'un modèle réduit, qui est souvent la seule solution pratique lorsque les études théoriques ou l'expérimentation en vraic grandeur sont impossibles.

\section{II. - LES PROBLEMES POSÉS PAR LE MOdËLE RÉduIT DE L'ESTUAIRE DE LA SEINE}

2.1 - On demandait au modèle de vérifier l'efficacité des ouvrages prévus par la loi de 1932, de préciser les conditions d'ouverture du nouveau chenal et surtout de montrer l'évolution des profondeurs dans un avenir de plusieurs dizaines d'années. 
Il fallait donc construire un modèle à fond mobile, permettant de contrôler l'évolution des profondeurs et la durée de stabilisation du nouveau chenal. Il fallait connaître l'échelle du «temps d'évolution des fonds » dont la détermination était le problème à la fois le plus inportant et le plus difficile.

Le modèle devait évidemment posséder les qualités de justesse, fidélité et sensibilité de tout véritable appareil de mesure.

2.2 - Les facteurs naturels du modelé des fonds sont nombreux et complexes (hauteurs et conrants de marée, houles, débit fluvial, matériaux de fond, salinité...). Les lois physiques de l'action des facteurs naturels sur les fonds étant incom- plètement connues, il était a priori difficile de déterminer les facteurs qui pouvaient être négligés et ceux dont la reproduction était indispensable : pour des raisons techniques et financières évidentes, certains facteurs (force de Coriolis, transports de vase, salinité) ont été négligés.

Enfin, le choix du matériau de fond a été déterminé par les essais du réglage préliminaire, mais rien ne permettait d'affirmer que la loi du transport solide variable pour le modèle représentait bien la nature.

3.3 - La construction du modèle réduit posait donc des questions très délicates aussi bien sur le plan théorique que pratique.

\section{III. - LA REALISATION DU MODELE RÉDUIT DE L'ESTUAIRE DE LA SEINE AU LABORATOIRE DE LA SOGREAH}

3.1 - Le modèle représente l'estuaire de la Seine entre le méridien de Villers-sur-Mer et Quillebeuf, à une échelle de $1 / 800^{\circ}$ en plan et $1 / 100^{\circ}$ en hauteur. L'échelle des temps hydrauliques est de 12 h $30 \mathrm{mn}$ nature pour $9 \mathrm{mn}$ modèle. Le matériau de fond était une sciure de bois assez fine (fig. 2).
3.2 - Les modèles réduits se justifient d'une manière théorique par l'application des règles de similitude aux équations des phénomènes naturels dont l'expression mathématique est connue, mais dont les conditions aux limites ou initiales sont complexes ou mal définies.

- Dans le cas de l'estuaire de la Seine, l'écou-

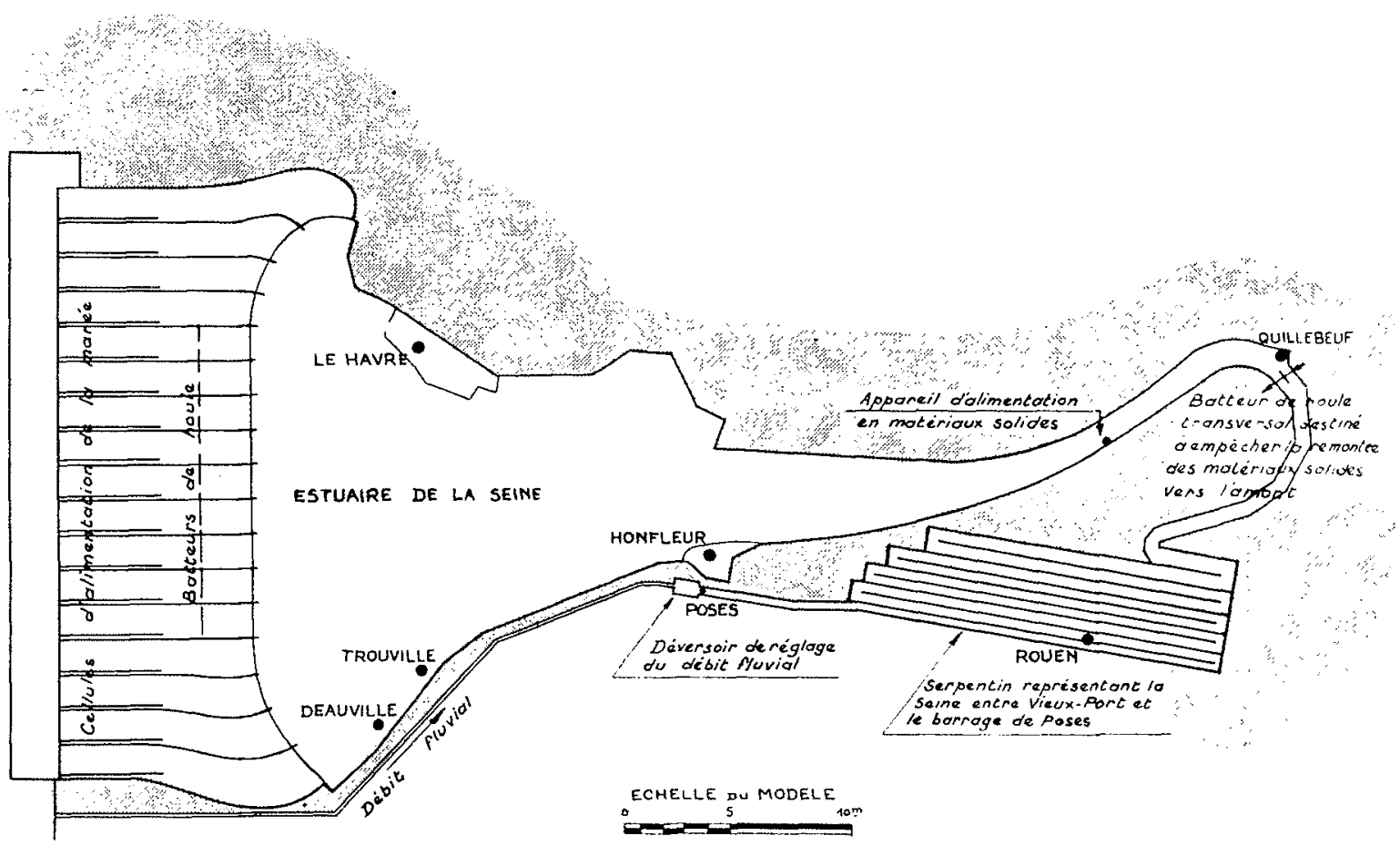

FIg. 2

Estuaire de la Seine. Plan schématique du modèle réduit de la SOGREAH.

Echelle du modèle : en plan, $1 / 800^{e}$ 
lement fluvial, la propagation de la houle et des marées ont permis de fixer l'échelle des temps hydrauliques $(t)$ en fonction des échelles de longueur en plan $(\lambda)$ et des hauteurs $(\mu)\left(t=\lambda \mu^{-1 / 2}\right)$.

-.. La condition de similitude globale de reproduction du modelé des fonds a permis, par la considération des lois de Lacey et de la « finesse » du modelé, de déterminer la distorsion.

- Des considérations théoriques sur le transport solide (théorie d'Einstein) et la similitude du frottement ont permis de reproduire l'ensemble des transports-nature par le seul charriagemodèle; mais aucune considération théorique n'a permis de fixer l'ćchelle des temps d'évolution des fonds, qui ne saurail être a priori celle des lemps hydrauliques.

3.3-La limite ouest du modèle comportait 17 cellules dont les débits étaient réglés pour reproduire la courbe de la marée de coefficient 95 au Havre; cette simplification considérable n'était cependant pas arbitraire et reposait sur la prépondérance de l'action des marées de forts coefficients, confirmée par les réglages préliminaires.
- La houle était également simplifice et son action limitée au rôle de catalyseur pour la mise en mouvement des matériaux qu'elle joue effectivement dans la nature. Le débit fluvial comportait l'équivalent d'une crue de $1200 \mathrm{~m}^{3} / \mathrm{s}$ pendant 9 heures-modèle et d'un étiage de $250 \mathrm{~m}^{3} / \mathrm{s}$ pendant 18 heures-modèle.

- Enfin, si le modèle reproduisait le phénomène caractéristique que constitue l'engraissement séculaire de l'estuaire à la cadence moyenne de 3 millions de $\mathrm{m}^{3}$ par an (1), l'alimentation en matériaux solides avait dû, pour des raisons techniques, être reportée en amont de la Risle, el ne respectait pas l'origine marine des apports solides de l'estuaire.

3.4 - On ne pouvait donc qu'être frappé à première vue par l'extrême simplification des phénomènes naturels reproduits sur le modele; en fail, les expérimentateurs ont bien compris qu'un réglage plus poussé que les réglages classiques étail nécessaire pour faire confiance aux résultats du modèle.

\section{I. - LES ESSAIS D'HISTORIQUE}

4.1 - Le réglage du modèle consistait à reproduire la situation des fonds, les lignes d'eau et les courants de 1947. Ces réglages ont permis de choisir le matériau de fond, de fixer à 8 la distorsion et de mettre au point la technique du modèle.

On ne pouvait cependant déduire de la reproduction d'une situation déterminée la valeur des critères utilisés pour ces réglages, ni l'échelle des temps d'évolution des fonds. En outre, la siluation de 1947 n'était pas stable, et la seule façon de vérifier si cette instabilité était un défaut ou une tendance à reproduire la nature, était de la suivre dans le temps et de la comparer avec des situations connues de la nature. Les essais d'historique étaient donc l'aboutissement naturel des réglages classiques.

4.2 - L'examen des positions du chenal dans l'estuaire de la Seine paraît à première vue assez anarchique; on peut cependant y trouver une périodicité de l'ordre de 18 ans (2) pendant laquelle le chenal passe d'un tracé sud à un tracé nord par l'intermédiaire d'un tracé central. Devait-on

(1) Ce phénomène traduit la tendance génémale des côtes découpées à «régulariser » leur tracé.

(2) Cette périodicité peut être rattachée à celle des «marées actives» établie par M. Gougenheim. limiler la durée de l'historique à un cycle de 18 ans?

En fait, le Port de Rouen, voulant vérifier l'efficacité des ouvrages pendant une cinquantaine d'années, il paraissait normal de demander une durée équivalente pour la reproduction du passé. Mais le Port de Rouen disposait de reconnaissances hydrographiques annuelles depuis 1864 : une période d'essai comprise entre 1875 et 1953 permettait donc d'étudier l'évolution d'un estuairc libre (1875-1895), puis l'effet des ouvrages d'anciens aménagements $(1895-1925)$ et à nouveau un estuaire libre en présence d'ouvrages anciens (1925-1953).

4.3 - Une première série d'essais historiques a été exécutée en 1955; elle a porté sur la période 1869-1953. Dès la mise en eau du modèlc, l'évolution des fonds a été rapide et le modèle a reproduit très correctement la nature. Des cubatures effectuées entre les années 1870 et 1890 ont fait ressortir un engraissement annuel du modèle correspondant à 5 millions de $\mathrm{m}^{3}$ et ont permis d'ajuster l'alimentation en matériaux solides.

Dans toute la période de 1870-1890, le modéle a reproduit les variations périodiques des chenaux, mettant en évidence un processus naturel d'évolution cyclique. 


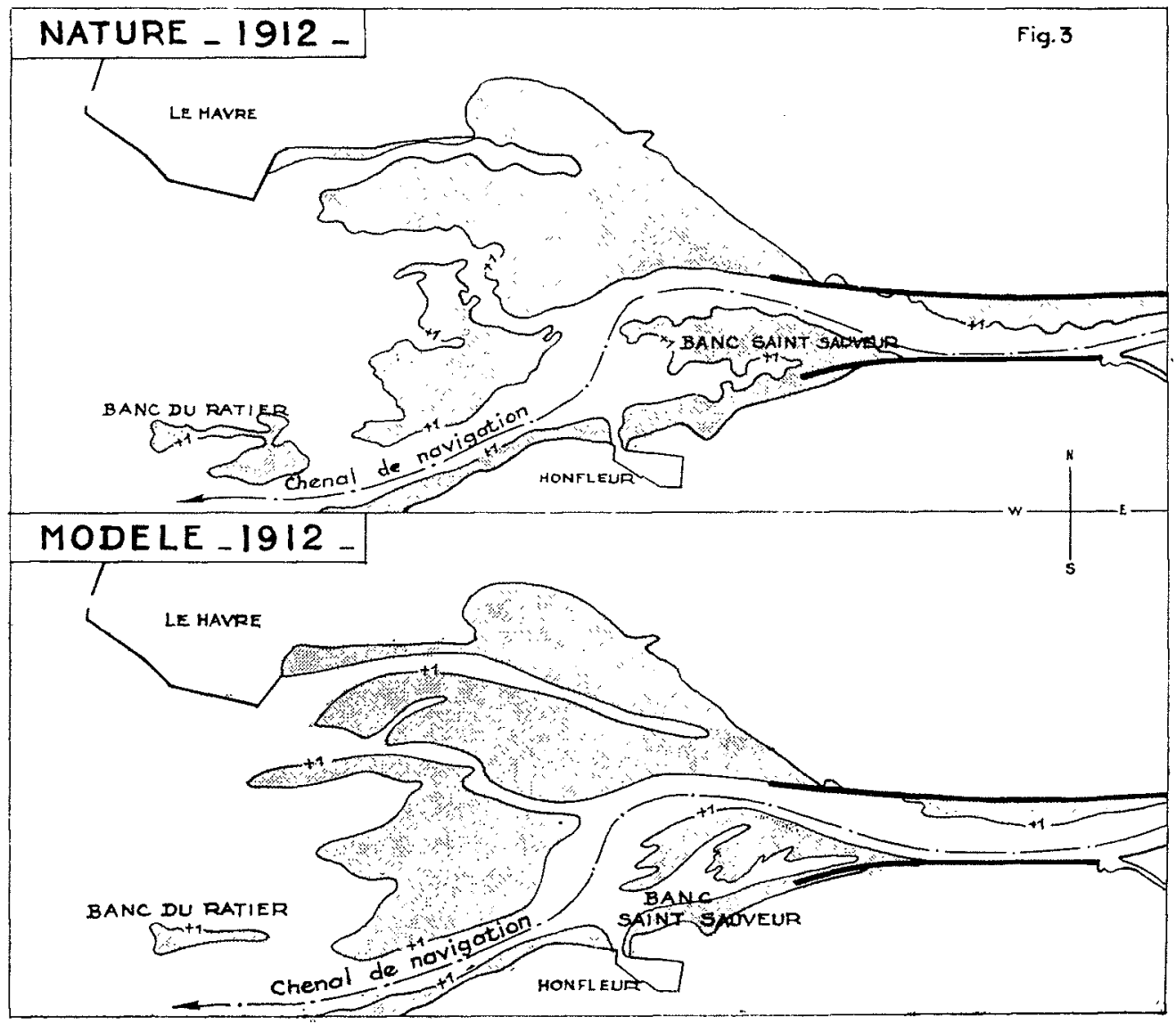

FIG. 3

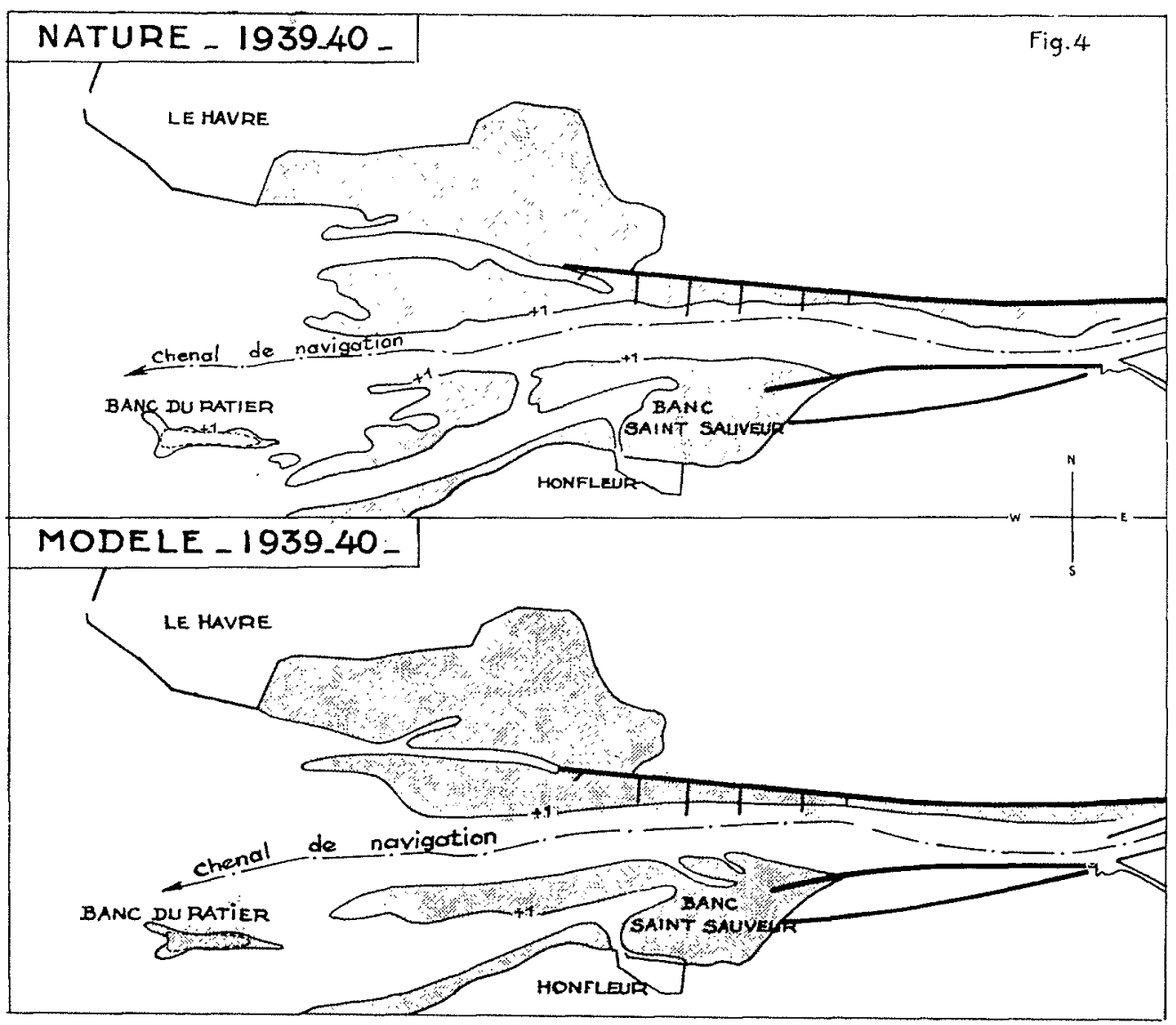

F1G. 4 


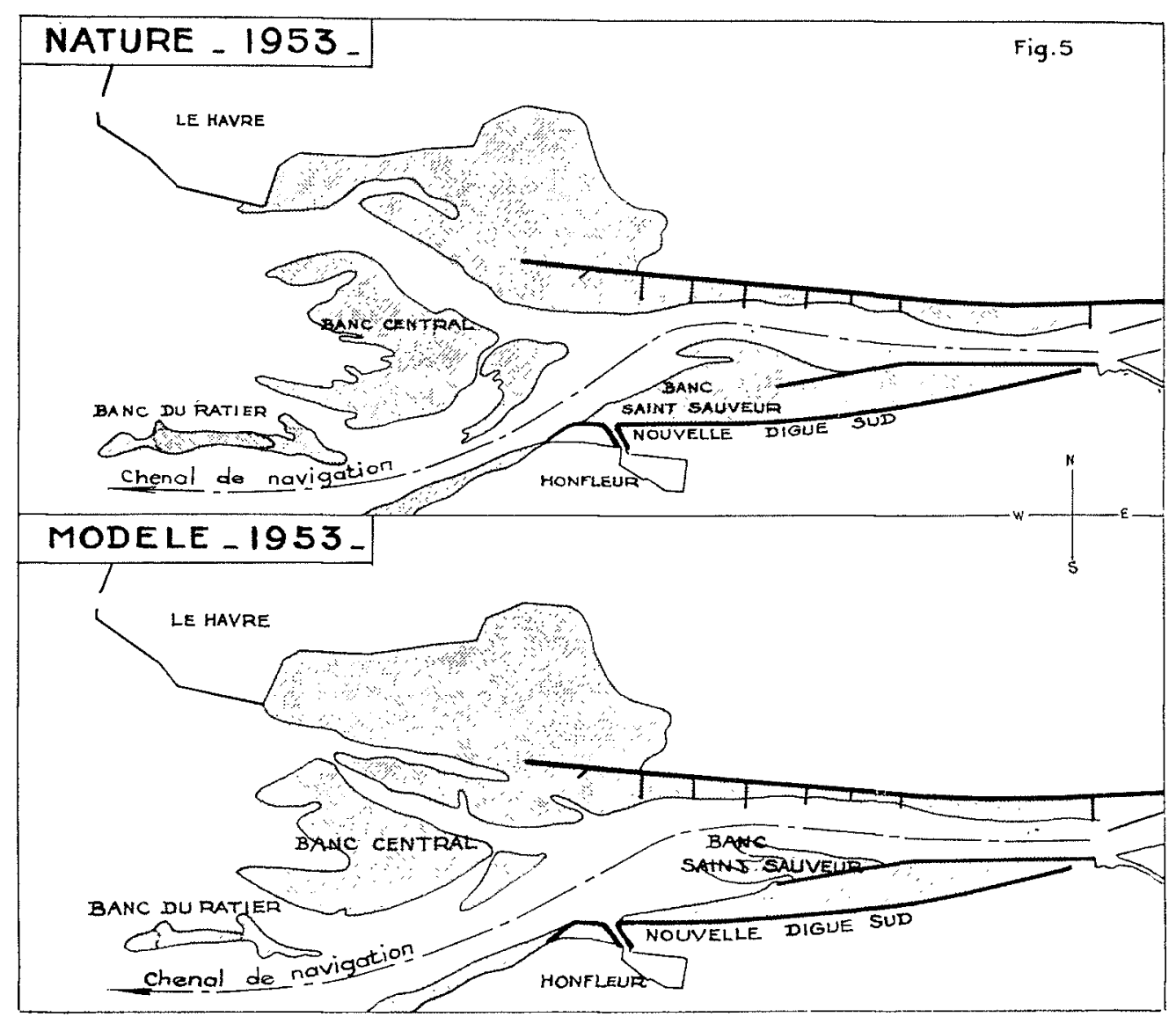

FIG. 5

La construction de la digue sud entre la Risle et la région d'Honfleur a provoqué, dès 19061907, la formation $d u$ banc de Saint-Sauveur dont le tracé en plan a subi diverses fluctuations reproduites par le modèle. Les épis de la digue nord construits entre 1923 et 1925 ont rejeté vers la digue sud le chenal qui s'était stabilisé contre la grande digue nord en amont du méridien d'Honfleur. Puis les fonds ont évolué vers un chenal central en 1939 pour se stabiliser au sud jusqu'en 1953 (fig. 3, 4, 5).

Il est intéressant de noter que le modèle a reproduit de facon régulière non seulement les évolutions globales des chenaux principaux et secondaires, mais aussi les « micro-évolutions », qui s'y retrouvent avec une grande fidélité.

4.4 - Trois ans après la première série d'essais historiques, une deuxième série fut entreprise en 1958, portant sur la période 1906-1922. Dès le début de cet essai, on constate la formation, puis le développement du banc de Saint-Sauveur; les inflexions du chenal se retrouvent suivant le même tracé et la même évolution dans le temps que celles de la nature.

4.5 - RÉsultats DE L'EsSai D'Historique. Les essais d'historique ont fourni le principal renseignement qu'on en attendait : l'échelle du temps d'évolution des fonds qui est de 10 heures-modèle par année nature, on 1 an-nature pour 60 marées-modèle. Les essais qui devaient suivre prenaient alors une valeur considérable, puisqu'on pouvait désormais connaître la duréc d'efficacité d'un ouvrage, celle de la période transitoire nécessaire à la stabilisation d'un chenal suivant un nouveau tracé, et la cadence d'exéculion des dragages. Le modèle était devenu un véritable appareil de mesure dont les essais avaient prouvé la grande justesse, malgré l'importante schématisation qui avait dû être consentie pour des raisons techniques et financières.

La fidélité est le résultat implicite des essais, puisqu'au cours des deux séries d'essais, le modèle a reproduit avec une remarquable coinncidence une longue période d'évolution des fonds.

Les essais d'historique ont également révélé la sensibilité du modèle, qui apparaît non seulement dans l'exactitude de la finesse du modelé, mais aussi dans certains incidents en cours d'essais. On a constaté, en effet, au cours de la deuxième série d'essais historiques, qu'aux environs de l'année 1914, contrairement à la nature, le chenal se fixait de façon très stable contre la digue nord et que la coupure du banc central s'amorçait difficilement. Un examen du modèle 
a révélé que la digue nord avait été prolongée trop en aval sur plus d'un $\mathrm{km}$. La suppression de cette longueur excédentaire a immédiatement entrainé la coupure du banc et une évolution conforme à la nature.

Le succès de l'essai d'historique a non seulement validé les simplifications admises pour la représentation des phénomènes, mais il a en outre justifié la valeur des conditions aux limites : la constance relative qui leur était imposée par l'obligation de reproduire la courbe de marée du Havre, n'a nullement entravé l'évolution naturelle des fonds à l'intérieur de l'estuaire.

Il importe cependant de souligner que le suc- cès de l'historique n'est pas un hasard heureux et que les réglages préliminaires permettaient d'aborder l'essai historique avec le maximum de chances de réussite; le choix du matériau de fond, en particulier, a été déterminé par la reproduction de la dégradation d'un chenal par détérioration simultanée de son débouché aval et de son inflexion au droit d'Honfleur. Ces éléments ont été déterminés avant l'exécution des essais historiques qui en ont sanctionné la valeur, sans introduction de nouvelles corrections au cours de ces essais. Le succès de l'historique était donc implicitement contenu dans les études et les réglages antérieurs.

\section{V. - CONCLUSIONS SUR L'INTÉRÊT DE LA METHODE HISTORIQUE}

La méthode historique permet donc de parfaire le réglage d'un modèle à fond mobile et de déterminer l'échelle des temps d'évolutions de fonds qui ne peut être calculée par des considérations théoriques, ni par un réglage en régine permanent.

Pour le modele de l'estuaire de la Seine, l'hislorique a validé les conditions aux limites et la reproduction des phénomènes naturels.

- L'historique a évidemment permis de « faire rationmellement $\gg$ confiance au modèle, car les essais des ouvrages de l'aménagement se sont déroulés sans modification de la technique des essais antérieurs. En outre, la notion de passé et d'avenir n'a sur le modéle qu'un sens assez conrentionnel; si nous appelons «présent» la situation de 1913, nous pouvons dire que le modele a reproduit correctement quarante ans du passé et quarante ans d'avenir. Il n'y a donc $a$ priori aucune raison pour refuser de transporter l'ori- gine des temps en 1953 et considérer que l'exactitude de quarante ans de passé permet de juger lefficacité des ouvrages projetés pendant une période d'égale durée dans l'avenir.

- Enfin, la méthode historique peut permettre de dégager des lois naturelles dans de meilleures conditions que l'observation des phénomènes en vraie grandeur, qui sont souvent déformés ou masqués par des interventions humaines ou des variations accidentelles.

- L'expérience du modèle de l'estuaire de la Seine montre que les essais d'historique sont l'aboutissement naturel de la longue campagne de mesures des phénomènes naturels et de minutieux réglages classiques; le cas de la Seine n'est d'ailleurs pas la seule réussite de la méthode historique, mais elle est particulièrement intéressante en raison de la grande duxée de son application et de la généralité de sa portée.

\section{IS CUSSION}

Président: M. DE RouviLLE

M. le Président félicite M. Chapon pour son exposí et pose à M. Govgenherm, Directeur du Service hydrographique, la question suivante :

Au point de vue de l'influence respective des années à nombreuses grandes marées, et des autres, quelle est, entre l'année Ininimale et l'année maximale, l'importance des totaux de coefficients, car il paraît que cet élément joue sur les déplacements des chenaux?

M. Govgenherm indique que l'effet énergétique des marées comporte une périodicité de 18 ans $2 / 3$ qui est celle de l'inclinaison du plan de l'orbite lunaire sur l'équateur, en raison de la rotation de la ligne des nœuds de la lune. De ce fait, le maximum de déclinaison de cet astre oscille entre 280 et $18^{\circ}$. Les ondes diurnes sont très sensibles aux variations de la déclinaison; les ondes semi-diurnes le sont beaucoup moins, leur coefficient étant proportionnel au carré du cosinus de la déclinaison, qui est donc compris entre 1 et un minimum allant de 0,77 à 0,90 . Si on limite la marée a l'onde prépondérante sur nos côtes, l'onde semi-diurne 
lunaire $M_{2}$, l'énergie annuelle de la marée varie au cours d'un cycle de dix-neuf ans comme le carré du coefficient astronomique de cette onde, soit de 0,93 à 1,08 .

Pour le déplacement des matériaux sous l'effet des courants de marée, le problème est beaucoup plus complexe, car il existe un seuil de vitesse au-dessous duquel le courant n'agit pas, seuil qui varie avec la nature du fond mobile considéré; en outre, le courant ne garde pas une direction constante et présente souvent un caractère alternatif rendant difficile la détermination du transport résiduel au cours d'une marée. L'existence du seuil de vitesse augmente l'importance des marées de fort coefficient et élimine plus ou moins celles de coefficient faible, de sorte que les annèes riches en coefcient élevés sont nettement plus actives que les autres. Le calcul reste à faire et il est loin d'être simple, même fait d'une facon approchée: il suppose en premier lieu que l'on puisse établir une corrélation entrc le coefficient d'une marée et le transport résiduel correspondant.

M. le Président remercie M. Gougenhem de son intervention et signale qu'il y a là un champ de vérification qui apprendra beaucoup de choses nouvelles. Un calcul pourrait être fait de la somme des carrés des coefficients de marées actives. Il rapproche ce phénomène de celui que l'on rencontre dans les attaques de côtes et où existe aussi un seuil au-dessous duquel les effets des vents et des mers sont négligeables.

M. Gougenheim note que M. Chapon procède par tranches de quarante ans qui concernent donc sensiblement deux cycles lunaires de dix-huit ans deux tiers, de sorte que l'effet de périodicité correspondant à ces cycles disparaît presque complètement dans les expériences du port de Rouen.

M. Chazy (Port Autonome du Havre) voudrait savoir si l'incident qui a fait représenter, sur le modèle, la digue nord trop longue de $1 \mathrm{~km}$, indique que la réalisation du prolongement de cette digue dans l'avenir pourra modifier la situation dans l'estuaire.

M. Chapon fait remarquer qu'il s'agit d'une perturbation dans le passé (essai de 1914) : dans cet essai, on avait, par erreur, construit la digue jusqu'à $1 \mathrm{~km}$ plus à l'aval. En fait, la digue nord, achevée en 1920-1922, a été prolongée, dans le réel, beaucoup plus en aval du point représenté dans l'essai de 1914. En la faisant de plus en plus loin, le chenal colle contre cette digue nord, puisque, en 1920, on avait un chenal nord qui partait de la digue nord et arrivait sous les murs du Havre.

Dans le futur, cette digue, qui dépasse les cotes des plus hautes mers connues, ne sera plus attaquée parallèlement par le chenal de navigation, car les travaux actuels sont faits de manière à avoir un chenal sud qui passe au droit de Honfleur par la digue du Ratier et débouche au plein milieu de l'estuaire. On a pu prouver que les modifications que l'on apportait à l'intérieur de l'estuaire sont pratiquement sans effet appréciable sur la partie extérieure.

M. le Président demande si un ingénieur du Laboratoire d'Hydraulique qui a fait le modèle n'a rien à mentionner.

M: Barallier (SO.GR.E.A.H.) répond qu'il n'a presque rien à ajouter, si ce n'est des considérations techniques. Après réglages d'un modèle sur des considérations théoriques approchées et expérimentales, la validité du modèle est basée sur des comparaisons morphologiques et sur la reproduction d'évolutions antérieures. Cette dernière méthode, en plus de la confirmation du mode opératoire choisi, présente non seulement un intérêt économique sur la rentabilité des aménagements, mais un intérêt technique. En effet, elle a permis de faire sur modèle des dragages correctifs indispensables pour juger l'érolution future dans la nature, où l'on infléchira les évolutions par ces dragages. On a d'ailleurs retrouvé sur modèle la localisation des zones critiques à draguer.

M. le Président remercie M. Barallier pour ces indications.

M. Chazy pose à M. Chapon une nouvelle question : au cas où l'on viendrait à effectuer des prélèvements de matériaux dans l'estuaire (banc du Ratier), y aura-t-il des répercussions notables sur le comportement du chenal ou, au contraire, l'enlèvement de certains matériaux pourrait-il être favorable?

M. Chapon répond que l'on pourrait enlever tout le banc du Ratier (5 à 6 millions de mètres cubes) sans changer l'évolution de l'estuaire.

I. le Président pense que c'est une opération grave que d'enlever ce banc, qui est le résultat de l'équilibre de la nature comme dans tous les estuaires.

M. Chapon indique que le banc du Ratier est un banc de cailloux inamovible et, sur la demande de $M$. le Président, que les bancs d'Amfard et du Ratier ne varient pas, sauf par grande tempête où leur crête peut être modifiée sur 10 ou $20 \mathrm{~cm}$. Dans une certaine mesure l'enlèvement partiel du Ratier peut être considéré comme une opération presque favorable, car il augmenterait la capacité de l'estuaire.

M. le Président fait remarquer que, depuis longtemps la Seine a été calibrée trop étroite et que les digues sud et nord construites à partir de 1895 n'ont guère rattrapé cette erreur.

M. Chapon ajoute que la Seine est calibrée trop étroitement depuis 1848 et que l'on calibre encore petit, puisque l'on est obligé de partir des sections d'amont qui déterminent la dimension d'entrée. Actuellement, on essaie de vaincre le manque d'eau, qui provient de l'étroitesse, par une régularité de l'ècoulement. Il est intéressant de reporter les brutales discontinuités, dommageables pour la tenue des fonds, le plus en aval possible, car sur" le méridien du Havre, complètement à l'extérieur de l'estuaire, les courants sont faibles (de l'ordre de 3 a 6 nouds au droit de Honfleur et 1 à 2 nœuds à $3 \mathrm{~km}$ en aval de Honfleur et inférieure à 2 nouds sur le méridien du. Havre). Les matériaux du fond d'origine marine seront ainsi faiblement mobilisés en aval, et on peut espérer qu'il n'en entrera pas beaucoup. Il en serait tout autrement si c'étaient des apports de l'amont.

M. le Président ne regrette pas d'avoir posé cette question à M. Crtapon, puisqu'il a fait ressortir la tendance du Service à déplacer la discontinuité sur une région plus favorable à l'aval.

M. Chazy fait remarquer qu'il pourrait y avoir des répereussions sur les zones d'accès du port du Havre si la discontinuité était reportée plus à l'ouest. Au cas où les travaux seraient poursuivis vers l'aval, de nouvelles études sont nécessaires montrant les inconvénients, les précautions à prendre.

M. le Président dit à M. Chapon que, dans la page 3 de sa communication, il néglige la force de Coriolis dans l'espèce.

M. Chapon indique qu'on a essayé de calculer l'effet de la force de Coriolis : très schématiquement, elle est à peu près équivalente à l'effet d'un courbure de l'ordre de $30 \mathrm{~km}$ de rayon. Dans l'estuaire, la force de Coriolis n'intervient pour ainsi dive pas.

Le projet actuel consiste à construire dans l'estuaire une berge prolongée par la digue du Ratier et à tracer une digue nord. Cette berge a une courbure de l'ordre 
de $30 \mathrm{~km}$. Si cette force de Coriolis double l'effet de la courbure pour le courant de flot, elle annule pratiquement la courbure pour le courant de jusant. Mais e'est nẻgligeable pour un phénomène qui intéresse une longueur de $10 \mathrm{~km}$ environ dans la partie indiquée et de $1 \mathrm{~km}$ dans la partie la plus large.

M. le Président indique que l'on trace toujours la digue du Ratier rectiligne, et estime qu'on aurait pu lui donner une légère courbure.

M. Chapon répond que, sur les essais, la digue droile a donné les meilleurs résultats. Dans la partie endiguée, les courants sont parallèles à l'axe du chenal; en aval de Honfleur, les courants ne sont plus parallèles aux digues, le phénomène est à deux dimensions et la courbure des digues en plan n'a pas le même effet que dans les parties étroitement endiguées.

M. BANAL voudrait faire des réserves sur la remarque de M. Chapon au sujet de la force de Coriolis. Il croit que le fait de comparer la force de Coriolis à un effet de courbure est sommaire et que le problème est plus complexe. Les avis sont partagés sur ce sujet et le problème est très mal connu. Cette idée simple, qui avait prévalu il $\mathrm{y}$ a quelques années, est très controuvée maintenant.

M. Chapon est tout à fait d'aceord avec M. Banal et confirme que la thèse actuelle du Port de Rouen relativement à cette question est la plus stricte neutralité ; il a précisé que le calcul était extrêmement sommaire et reposait sur des considerations théoriques trop schématiques pour etre valables (1).

M. le Président indique que l'incidence de la force de Coriolis dans les usines a marée et les plaques tournantes réalisées pour l'étudier dans ce domaine, permettront de reprendre la question du point de vue purement scientifique.

M. le Président termine cette discussion par deux réflexions qui lui sont venues :

10 La chance du Port de Rouen est de posséder des archives bien tenues depuis plus de cent ans : cela a permis de reconstituer en laboratoire l'histoire de l'estuaire, ce que ne peuvent faire les pays neufs;

20 Depuis quelques années les études sur la Seine ont été faites par des commissions; mais, maintenant, on a le modèle, qui coûte plus cher que les commissions, mais qui renseigne davantage et fait faire des économies sur la réalisation.

M. le Président remercie M. CHapoN et lui souhaite bonne chance pour la suite de ces travaux (applaudissements).

(1) On peut rappeler l'article de M. R. ABbort, publié par La Houille Blanche, $n^{\circ} 5$, de scptembre-octobre 1960 , relatif à l'elfet de la force de Coriolis sur la propagation de la narée dans un estuaire. Cette étude thérique prouve que 'effet de la force de Coriolis est négligeable pour un estuaire comme celui de la Tamise, en raison de ses faibles dimensions. Or, les caractéristiques de l'embouchure de la Tamise sont comparables à celles tle l'estuaire de la Scine.

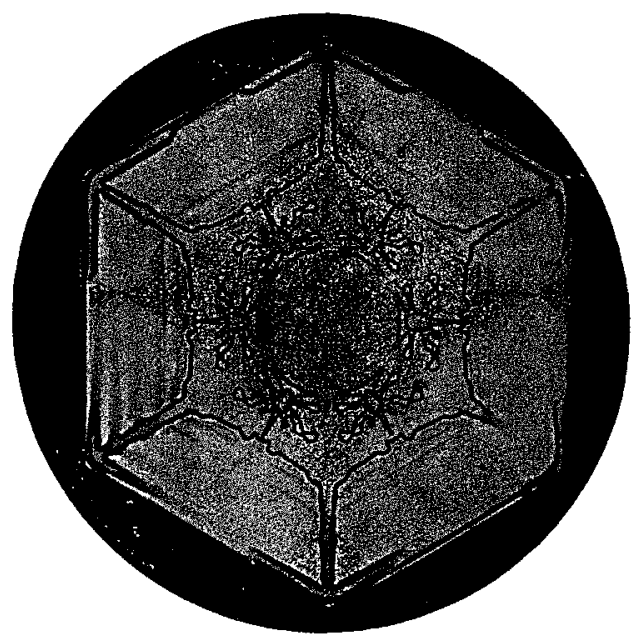

